\title{
Comorbid pain related somatisation is common in Latin Americans with major depressive disorder
}

Munoz RA, McBride ME, Brnabic AJM, et al. Major depressive disorder in Latin America: the relationship between depression severity, painful somatic symptoms, and quality of life. J Affect Disord 2005;86:93-8.

How prevalent is pain related somatisation in Latin Americans with major depressive disorder?

\section{METHODS}

$\square-$

Design: Cross sectional study.

(3)

Setting: Eighty centres in Argentina, Brazil, Chile, Colombia,

Mexico, Peru, Venezuela; time frame not specified.

ofe Population: 989 outpatients aged over 18 years old, with current, new, or first episode major depressive disorder (DSM-IV or ICD-10) not exceeding two continuous years duration. Participants were depression free for at least two months before their current episode. People with previous or current diagnose of schizophrenia, schizoaffective disorder, bipolar disorder, or dementia were excluded, as were those who were prescribed a monoamine oxidase inhibitor, had received electroconvulsive therapy, or had treatment resistant depression.

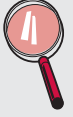

Assessment: The Somatic Symptom Inventory (SSI) was used to classify somatisation. Depression symptoms were measured using the 17-item Hamilton Depression Rating Scale $\left(\mathrm{HAMD}_{17}\right)$. The Quality of Life in Depression Scale (QLDS) measured quality of life.

Fise

Outcomes: Pain related somatisation (mean score $\geqslant 2$ on pain related items of SSI), symptoms of depression, quality of life.

\section{MAIN RESULTS}

Seventy three per cent of all people with major depressive disorders (717/987) reported painful somatic symptoms. Women were more likely than men to have pain related somatic symptoms (OR 2.7, 95\% CI 1.96 to $3.72 ; \mathrm{p}<0.0001)$. Muscle soreness, headache, and neck pain were the most prevalent symptoms $(82 \%, 78 \%$, and $78 \%$ respectively). People with painful symptoms had significantly greater depression severity and reduced quality of life compared with people with no painful symptoms (mean difference in $\mathrm{HAMD}_{17}: 3.92,95 \%$ CI 2.99 to $4.85 ; \mathrm{p}<0.0001$, mean difference in QLDS: $3.29,95 \%$ CI 2.19 to $4.39 ; \mathrm{p}<0.0001$ )

\section{CONCLUSIONS}

Many Latin Americans with major depression also experience painful somatic symptoms. Presence of these symptoms is associated with increased severity of depressive symptoms and reduced quality of life.

For correspondence: Margaret E McBride, mcbride_peggy_e@lilly.com Source of funding: Eli Lilly and Company.
NOTES

These data represent pooled baseline data from two prospective, cohort studies.

\section{Commentary}

7 his brief report describes the relation between depression severity, painful somatic symptoms, and quality of life in Latin American outpatients diagnosed with major depressive disorder. The large sample was recruited from study sites across seven Latin American countries. Reported ethnicity was varied: $38.1 \%$ white, $50.7 \%$ Hispanic, and the remaining $11.2 \%$ were African, Asian, mixed race, or "other" The study was well designed and the authors used reliable and valid diagnostic instruments and other assessment tools, and utilised solid analytic techniques. However, the lack of description of the study sites, such as the percentage of urban versus rural sites or primary care versus speciality care limits conclusions about generalisability.

The findings were mostly what one would expect: women were more likely than men to report painful bodily symptoms, such as muscle soreness, neck pain, and headache, and those who reported higher pain levels were more likely to endorse worse depression severity and worse quality of life.

The most interesting finding is the lack of a statistically significant relation between ethnicity and painful somatic symptoms associated with depression. This finding differs from other research showing that somatic presentations of depression are more likely to be observed in Latino patients compared with white patients. ${ }^{12}$ Further, this finding contrasts sharply with the training of physicians in the United States, who are frequently instructed to consider the possibility of underlying depression in Latinos presenting with medically unexplained physical symptoms. ${ }^{3}$

The current results are supported by other large international studies, which have also found no evidence for a culture specific phenomenon regarding greater emphasis of somatic symptoms in depression. ${ }^{4}$ Painful somatic complaints are extremely common in people with depression and negatively impact quality of life. Munoz et al's findings caution against stereotyping Latinos with depression as being more prone to somatisation.

Stacey Hart PhD

Department of Psychiatry, University of California San Francisco and the San Francisco Veteran's Affairs Medical Center, San Francisco, CA, USA

1 Canino IA R-SM, Canino G, Escobar Jl. Functional somatic symptoms: a cross-ethnic comparison. Am J Orthopsychiatry 1992:62:605-12.

2 Varela RE VE, Sanchez-Sosa JJ, Riveros A, et al. Anxiety reporting and culturally associated interpretation biases and cognitive schemas: a comparison of Mexican, Mexican American, and European American families. J Clin Child Adolesc Psychol 2004;33:237-47.

3 Lewis-Fernandez R, Das, AK, Alfonso C, et al. Depression in US Hispanics: diagnostic and management considerations in family practice. J Am Board Fam Pract 2005; 18:282-96.

4 Simon G GR, Kisely S, Piccinelli M. Somatic symptoms of distress: an international primary care study. Psychosom Med 1996;58:481-8. 